Etnográfica

Revista do Centro em Rede de Investigação em

Antropologia

vol. $23(3)$ | 2019

Vol. 23 (3)

\title{
Entre solidarité standardisée et solidarité renégociée : (re-)présentations de dettes dans un programme de microcrédit argentin
}

Between standard solidarity and renegotiated solidarity: (re)presentations of debts in an Argentinean microcredit program

\section{Bárbara Galarza}

\section{CpenEdition}

\section{Journals}

Édition électronique

URL : https://journals.openedition.org/etnografica/7170

DOI : 10.4000/etnografica. 7170

ISSN : 2182-2891

\section{Éditeur}

Centro em Rede de Investigação em Antropologia

Édition imprimée

Date de publication : 1 octobre 2019

Pagination : $557-578$

ISSN : 0873-6561

Référence électronique

Bárbara Galarza, «Entre solidarité standardisée et solidarité renégociée : (re-)présentations de dettes dans un programme de microcrédit argentin », Etnográfica [En ligne], vol. 23 (3) | 2019, mis en ligne le 27 novembre 2019, consulté le 20 janvier 2022. URL : http://journals.openedition.org/etnografica/ 7170 ; DOI : https://doi.org/10.4000/etnografica.7170

\section{(c) (†) 8}

Etnográfica is licensed under a Creative Commons Attribution-NonCommercial 4.0 International License. 


\section{Entre solidarité standardisée et solidarité renégociée : (re-)présentations de dettes dans un programme de microcrédit argentin}

\section{Bárbara Galarza}

Partant d'observations ethnographiques, cet article analyse les tensions qui traversent les perspectives mises en jeu dans un programme de microcrédits géré par l'État national dans la région pampéenne de l'Argentine pendant la première décennie du XXIe siècle. L'approche privilégiée fut de dénaturaliser la notion standardisée de la solidarité reproduite par les promoteurs de ce programme. L'étude des relations interpersonnelles avec lesquelles la dynamique du microcrédit se développe montre qu'elle comprend des pratiques de donner-recevoir-rendre, mais aussi celles de prendre-garder ou de refuser-à-rendre. Les bénéficiaires voient le temps qu'ils ont accordé au programme comme substituant leur obligation de rembourser leur prêt. De sorte, les pratiques qui s'avèrent « non solidaires » aux yeux des promoteurs sont ici réinterprétées comme une forme de valorisation native axée sur un échange de temps contre l'argent.

MOTS-CLEFS : solidarité, don, microcrédit, ethnographie.

Between standard solidarity and renegotiated solidarity: (re)presentations of debts in an Argentinean microcredit program - This article seeks to analyze, from an ethnographic point of view, the local dynamics in an Argentinean microcredit program administered by the national government in the Pampean region during the first decade of the $21^{\text {st }}$ century. The study of interpersonal relationships between promoters and beneficiaries shows that microcredit dynamics develops through practices of both giving-receiving-returning and keeping-rejecting-to-giveback. This is because beneficiaries evaluate the time given to the program as a substitute for the credit they are required to reimburse. I assert that these practices do not make up opposing logics but relational dynamics that motivate a renegotiation of the terms of exchange. The study shows that some cases and events that are considered "failures" from the point of view of the promoters of the program are experienced as mutual aid practices from the point of view of beneficiaries. By contrasting these perspectives regarding solidarity, I intend to show that "failures" in the program are also the expression of a native form of valorization based on the exchange of time for money.

KEYWORDS: solidarity, don, microcredit, ethnography.

GALARZA, Bárbara (barbaragalarza@gmail.com) - Universidad Nacional del Centro de la Provincia de Buenos Aires, Conicet, Argentina. 


\section{INTRODUCTION}

Araceli est une aimable ancienne employée d'une compagnie téléphonique qui habite un petit village de la région pampéenne argentine comportant à peine une dizaine de commerces. ${ }^{1}$ Âgée de 63 ans et retraitée, elle vit avec son fils adolescent dans une maison dont elle est propriétaire. Elle a aussi deux autres fils de 38 et 42 ans. Veuve, elle touche à une double pension (de sa propre retraite et de son mari décédé). Elle augmente aussi ses revenus par la revente de vêtements venant d'Inde à partir de son domicile. Sa participation à la vie communautaire et aux activités organisées par l'association de retraités qu'elle coordonne lui assure un large réseau de connaissances personnelles et un marché pour ses biens. C'est en 2011 qu'elle a fait l'heureuse découverte de la possibilité de demander un prêt qui lui permettrait d'augmenter son inventaire sans passer par la banque officielle ni devoir mettre sa maison ou sa voiture en gage comme garantie. Selon le représentant du gouvernement, la principale condition pour pouvoir obtenir ce crédit était de trouver quatre autres personnes disposées à demander leurs prêts individuels de manière collective. C'est ainsi qu'Araceli s'est mise à la recherche de candidats.

Marie est une coiffeuse agile et plutôt nerveuse âgée de 45 ans. Récemment séparée après un mariage de 20 ans, elle a deux enfants préadolescents à sa charge. Son mari, me dit-elle, ne lui donne pas d'argent et elle n'est pas éligible pour l'allocation universelle pour les enfants (AUH) à cause de son « bon salaire », soit l'équivalent de six salaires minimums. ${ }^{2}$ Ses tâches quotidiennes sont réparties entre divers emplois informels : soignante à domicile pour personnes âgées, masseuse, coiffeuse et femme au foyer. Contrairement à la cordialité d'Araceli, une certaine frénésie teignait l'attitude de Marie, qui se montrait toujours anxieuse et préoccupée par l'argent. Elle en avait besoin, non seulement pour « entreprendre » ou «faire croître » son « salon de coiffure $»^{3}$ mais aussi pour résoudre les conséquences économiques de sa récente rupture et « joindre les deux bouts ». Le crédit, disait-elle, l'aiderait à payer quelques factures, jusqu'à ce qu'elle obtienne un emploi plus fixe et stabilise sa situation financière. Elle avait déjà essayé de demander un crédit, mais cette année-là le ministère n'avait pas d'argent et elle trouvait qu'elle avait « perdu beaucoup de temps en allant à toutes ces réunions ». Or, en manque d'argent,

I Cet article est basé sur une communication présentée au colloque «Dynamiques coopératives et mutualistes » (tenu à Besançon en 2013), intitulée « De la pauvreté à l'entrepreneuriat engagé, le cas du microcrédit argentin ». La recherche a été réalisée grâce à la bourse Victor Hugo du Conseil régional de Franche-Comté et l'Université de Franche-Comté. Je voudrais remercier Julien Landry pour sa révision linguistique du texte et ses commentaires.

2 L'AUH fut créée en 2009 par le gouvernement argentin pour garantir une modeste somme d'argent à tous les enfants entre 12 semaines et 18 ans dont les parents sont au chômage ou dans le marché du travail informel.

3 J'utilise les guillemets pour rapporter expressions des documents officiels et du discours des acteurs. 
elle se remit, malgré tout, à chercher des gens avec qui elle pourrait demander du crédit.

Araceli et Marie sont les leaders de leurs groupes solidaires respectifs dans le cadre du même programme de microcrédit argentin. La première est considérée comme un cas de réussite selon les règles du programme. Son groupe a pu rembourser leurs prêts dans les formes et délais prescrits et a depuis reçu deux prêts supplémentaires (appelés « recrédits ») pour des montants plus élevés. En contraste, le groupe de Marie est considéré comme un échec complet, car il a échoué à retourner l'argent emprunté. Certains de ses membres ont d'ailleurs disparu dès qu'ils ont touché l'argent, devenant ainsi de mauvais bénéficiaires aux yeux des promoteurs. Or, en reconstruisant les perspectives des promoteurs et des bénéficiaires et en analysant leurs enjeux, l'étude de ces deux expériences met à jour les processus de construction d'obligations réciproques qui peuvent survenir dans un programme de microcrédit. ${ }^{4}$

Mon intérêt pour « les échecs » découle non seulement du nombre important de ces « insuccès », mais aussi du problème qu'ils laissent entrevoir. Les tensions entre les promoteurs et les bénéficiaires du microcrédit argentin indiquent que ces « échecs » ne sont pas réductibles à une « simple erreur» dans « la mise en œuvre de la méthodologie Grameen », comme le suggèrent les coordinateurs du programme. ${ }^{5}$ Dans leur rôle bureaucratique, ces derniers intimaient aux promoteurs de « respecter minutieusement les règles du programme lorsqu'ils accompagnent les personnes pauvres qui demandent du crédit afin de garantir le succès du programme selon les critères du ministère responsable ». Mais, dans leur rôle d'intervenants sur le terrain, les promoteurs furent confrontés chaque semaine à des problèmes qu'ils ne pouvaient résoudre en ayant recours qu'aux règles du programme. Alors qu'une de leurs principales fonctions était l'encaissement des remboursements, ils observaient que les gens avaient vraiment des difficultés à retourner l'argent, peu importe la méthodologie mise en œuvre. Ils étaient également perplexes devant la «trahison » des bénéficiaires qui quittaient le programme sans rembourser leur prêt : « pourquoi ne sontils pas solidaires comme le programme de microcrédit le propose ? » À titre de chercheure, je me demandais moi-même : les acteurs qui abandonnent le programme sont-ils réellement peu solidaires ? S'opposent-ils vraiment à la réciprocité du don quand ils ne retournent pas l'argent emprunté ? J'ai donc prêté une oreille attentive aux représentations natives de la dette pour essayer de comprendre comment l'échange de crédit et de dette est vécu et interprété par les bénéficiaires elles-mêmes.

4 Les observations exposées font partie d'une recherche dont l'objectif fut d'examiner les processus culturels qui ont contribué à l'adoption ou non de programmes de microcrédit en Argentine pendant les années 2000.

5 La méthodologie Grameen, établie par la Grameen Bank au Bangladesh, est le modèle pour l'application du microcrédit privilégié par l'État argentin. 
L'ethnographie du non-remboursement de dettes peut offrir des clés interprétatives pour mieux comprendre la constitution socioculturelle des dits « échecs » du programme de microcrédit d'un point de vue natif. Tout comme Pierre Bourdieu s'était rendu compte qu'il n'y avait rien de « naturel » dans l'habitus économique capitaliste que les Français essayaient d'imposer à la société kabyle (Bourdieu 2003 [1967]), je pense qu'il n'y a rien qui va de soi dans la «solidarité » que les cadres de l'État s'attendent à retrouver ou générer chez les classes populaires auprès desquels ils interviennent. Dans le but de dénaturaliser les rapports « solidaires » instrumentalisés par les États nationaux qui mettent en œuvre les programmes de microcrédits, je mets l'accent sur les cas dans lesquels l'argent est une ressource rare. Je procède en comparant les pratiques technocratiques de construction de la solidarité groupale (appelées « les sept pas ») aux conceptions du remboursement de dettes avancés par les bénéficiaires qui n’ont pas retourné le crédit emprunté. Le contraste m'induit à risquer que la représentation de l'aide mutuelle constitue un enjeu qui comporte la possibilité de rompe avec le répertoire de la solidarité évoquée par les promoteurs. Vues de cette manière, les femmes mobilisent des liens de solidarité qui correspondent aux ressources qu'elles peuvent effectivement rendre. De ce fait, elles produisent des manières de concevoir leur dette qui diffèrent de celles anticipées par le programme. En particulier, elles envisagent de substituer la dévolution de l'argent pour celle d'une autre ressource - leur temps. Dans ces échanges, elles réussissent à solder leurs dettes et à entretenir des relations solidaires en leurs termes, mais des tensions surviennent lorsque cette logique se confronte à celle des cadres de l'État.

Mes recherches furent effectuées dans trois localités - de 5000, 65.000 et 100.000 habitants - de la province de Buenos Aires, Argentine, entre avril 2011 et février 2012. Géographiquement, le territoire se place dans une zone d'exploitation agricole, d'élevage intensif de bétail et de production industrielle. J'y ai effectué des observations participantes : de manière hebdomadaire au sein de réunions de bénéficiaires et de manière mensuelle dans des réunions entre les coordinateurs et les promoteurs. Au total, j'ai étudié 15 bénéficiaires qui appartiennent à trois groupes de demande de crédit différents, ainsi que cinq promoteurs.

Le terme « bénéficiaire » désigne la personne qui prend le crédit octroyé par l'État. Le « promoteur» est l'employé de l'État chargé d'exercer un contrôle comptable sur les activités économiques présentées lors de la demande de crédit. Il est aussi intimé d'encourager le remboursement du prêt et la participation des groupes dans diverses activités collectives. Les coordinateurs sont surtout des travailleurs sociaux qui entretiennent des liens étroits avec le gouvernement municipal. ${ }^{6}$ Ceux-ci sont des agents supra-locaux qui

6 Plusieurs d'entre eux réussissent à faire carrière comme conseillers locaux, dans le législatif, ou à la mairie, dans le secrétariat au développement social. 
participent rarement aux activités quotidiennes de la demande et de l'octroi du crédit. Leur rôle dans le fonctionnement quotidien du programme est principalement bureaucratique, puisqu'ils font le pont entre les promoteurs locaux et l'État. Les promoteurs doivent remplir un formulaire pour rapporter l'information personnelle et les activités économiques des bénéficiaires. Un coordinateur réunit ces informations et les transmet au Ministère du développement social. Les officiers du ministère déterminent ensuite la disponibilité de fonds publics pour ces crédits en fonction d'une évaluation de la performance régionale des banquitos (petites banques), c'est-à-dire des associations créées dans le cadre des politiques sociales de l'État et décrites avec ce diminutif espagnol pour dénoter leur caractère informel, à la différence des « vraies banques formelles $» .^{7}$

\section{LE CONTEXTE GLOBAL ET LOCAL DU MICROCRÉDIT}

Un grand nombre d'études récentes s'intéressent au phénomène de la financiarisation «par le bas» (Guérin 2015; Morvant-Roux et al. 2014) et surtout à la crise du microcrédit qui en découle (Guérin, Labie et Servet 2015). Dans « La microfinance et ses dérives : émanciper, discipliner ou exploiter? », Isabel Guérin caractérise les principales perspectives sur le microcrédit. Elles traversent les disciplines académiques, incarnant une vision du développement enchantée par la transformation des pauvres en de «vrais entrepreneurs ». En même temps, ces pauvres deviennent « une niche de marché à exploiter pour les banques commerciales » (Guérin 2015: 15). Au niveau local, on observe que le microcrédit adopte une diversité de formes, allant des dispositifs dits «populaires » à ceux qui sont administrés de manière « purement financière » (Guérin et Servet 2005: 83). Les rapports de dettes que génèrent ces programmes peuvent être à la fois source d'exploitation, d'autonomisation et de réciprocité. Mais ce que ces dispositifs ont en commun, c'est qu'ils utilisent le discours de la « solidarité » comme outil idéologique pour assurer leur constitution et leur diffusion.

Le cas argentin du microcrédit que l'on présente ci-dessus constitue une expérience de type populaire, c'est-à-dire conçue comme un projet sociocommunautaire plutôt que commerciale. Il a récemment été étudié comme une expérience de politique publique de gauche qui a «priorisé les considérations politiques sur la viabilité économique », résultant en « une politique partisane qui empêchait d'aider les pauvres » (Ahnen 2017: 58). Or, une recherche ethnographique décrivant la perspective des bénéficiaires, ainsi que celle de l'État, peut mettre en lumière certaines dimensions qui demeurent inexplorées

7 En fait, pour pouvoir fonctionner, ces banquitos dépendent d'autres institutions communautaires qui leur prêtent leurs installations. 
par les approches axées sur le développement et l'action publique. Dans la littérature anthropologique argentine, les études du programme du microcrédit ont porté sur sa construction comme projet politique, pratique économique et mythique (Koberwein 2011, 2012).

Le Banco Popular de la Buena Fé (Banque Populaire de la Bonne Foi, dorénavant $\mathrm{BPBF}$ ) est le produit d'une politique sociale formulée et gérée au niveau national par le Ministère du développement social argentin et mis en place par des associations locales. Il s'agit d'un programme de microcrédits qui prête de l'argent à un taux d'intérêt peu élevé ou même nul, à des personnes « exclues du système bancaire formel ». Le BPBF dépend de la Commission nationale du microcrédit. ${ }^{8}$ Ses objectifs principaux incluent :

«le soutien et l'accompagnement des entrepreneurs, l'organisation populaire et la construction de liens de confiance et de solidarité entre les travailleurs et les organisations sociales. En effet, le microcrédit est beaucoup plus qu'un petit prêt. C'est une opportunité pour que des milliers d'Argentins développent de manière associative ou familiale des projets de production, de livraison de service et de vente au détail $\gg .{ }^{9}$

Suivant le modèle de la « Grameen Bank » du Prix Nobel de la Paix Muhammad Yunus, ce programme fut créé en octobre 2002 comme une forme d'aide aux populations démunies. L'existence d'une énorme population de chômeurs sans couverture sociale et l'émergence de logiques coopératives ne sont pas des phénomènes nouveaux en Argentine. En effet, des instances mutualistes introduites par les immigrants européens à la fin du XIXe siècle se multiplièrent durant la première moitié du XXe siècle au sein d'associations d'aides mutuelles (Socorros Mutuos) dont la cohésion dépendait de rapports identitaires espagnols, polonais, français et italiens. ${ }^{10}$ Pendant la deuxième moitié du XXe siècle, le nombre de ces associations a diminué avec l'expansion du travail industriel salarié et la création de services de santé et d'éducation garantis par l'État. Un demi-siècle plus tard, soit au cours de la première décennie des années 2000, le pays fut confronté à une crise sociale et économique renouvelée. Avec des taux de pauvreté de 57,5\% et d'indigence de 27,5\%, l'État devait composer avec une masse d'hommes sans emploi dont la «culture du travail » était, croyait-on, « en danger». Cette situation exacerba aussi la vulnérabilité sociale des épouses et des filles de ces chômeurs. Plusieurs de ces femmes participèrent

8 La Conami (Comisión Nacional de Microcrédito) fut créée par la Loi N. ${ }^{\circ} 26.117$ et fait partie du Ministère de la santé et du développement social.

9 Page officielle du Ministère de la santé et du développement social argentin, < http://www.desarrol losocial.gob.ar/conami/120 > (dernière consultation en octobre 2019).

10 En Argentine, l'association mutualiste inscrite la plus ancienne est l'Union de secours mutuels de l'Association française, fondée le ler octobre 1854 par des immigrés français. 
au programme de la BPBF pendant la décennie 2000-2010 et elles y participaient encore au moment de mon travail de terrain en $2011-2012 .{ }^{11}$

En aval de la crise politique et économique nationale des années 2000, la BPBF a offert des formations, des appuis et des fonds à plus de 1500 associations travaillant auprès d'organismes provinciaux et municipaux. Les conditions pour accéder aux prêts administrés par la BPBF et son réseau d'associations sont assez simples : il faut être majeur (18 ans), être chômeur ou sous-employé (ce qui n'est pas vérifié) et ne pas avoir accès au crédit formel. Il est ensuite espéré que ces bénéficiaires puissent contribuer à la croissance du programme en faisant fructifier leur crédit et en remboursant leurs prêts.

Certains auteurs affirment que la microfinance est un mouvement globalisé conforme à la même logique néolibérale à laquelle elle s'oppose prétendument (Servet 2006 ; Fouillet et al. 2007). Selon eux, le discours pour dépasser la pauvreté qu'elle reproduit contribuerait à renforcer le mythe néolibéral de «l'homo entrepreneur ». En s'associant à l'État, cette logique produit une nouvelle manière de gérer les politiques sociales, s'arrimant au modèle de « l'État qui fait faire »(Guérin et Servet 2005: 84). Dans cette perspective, je propose d'observer et d'analyser le microcrédit à l'échelle locale pour mieux comprendre comment l'État « fait faire » et comment les acteurs font effectivement avec ce qu'ils ont.

Je considère donc la BPBF comme un référent ethnographique pour l'étude anthropologique d'une «pratique économique » qui se dit «solidaire». Cette recherche considère, d'une part, la perspective des techniciens du programme (principalement en ce qui a trait aux contradictions auxquels ils sont confrontés dans la gestion quotidienne de la méthodologie Grameen) et, d'autre part, la perspective de groupes de femmes organisées dans le but de solliciter les microcrédits. Afin d'illustrer les conceptions et les pratiques natives dans le processus d'acquisition du microcrédit, mon registre ethnographique focalise sur deux femmes qui furent les leaders de leur groupe solidaire respectif. L'observation des pratiques qui ont structuré les interactions au sein de leurs groupes respectifs suggère une hétérogénéité des représentations de ce que constitue le « succès » ou «l'échec » dans le cadre du programme de microcrédit, notamment par rapport au délai du retour de la dette et au moyen par lequel celle-ci est considérée comme étant remboursée. Il fut donc utile d'analyser ces interactions à la lumière d'une conception anthropologique du don et de la dette.

11 Le phénomène de l'économie populaire s'est répandu en Argentine pendant les années 2000 en raison de la croissance de la pauvreté. La multiplication du troque découle de l'approfondissement du travail informel (Saiag 2011). Le sujet du crédit informel entre femmes au chômage a été étudié dans les quartiers au sud-ouest de la ville de Buenos Aires (Schijman 2014). 


\section{L'ANALYSE DU MICROCRÉDIT COMME EXPÉRIENCE VÉCUE DE DON ET DE DETTE}

Franz Boas et Marcel Mauss se sont servis de la métaphore du crédit pour caractériser et contraster le fonctionnement des échanges dans les sociétés du Nord-ouest américain (voir Mauss 2007 [1925]: 137-138). Florence Weber a prolongé ces études en analysant le crédit non pas comme une métaphore, mais à partir des interactions sociales qui le sous-tend : «tandis qu'un contrat de crédit fonde l'unité de tous les transferts compris entre l'ouverture du crédit et son extinction, un échange de dons consiste explicitement en deux transferts distincts l'un de l'autre dont le second ne clôture pas la relation ouverte par le premier » (Weber 2007: 28-29).

En effet, les microcrédits de la BPBF se déroulent en de multiples étapes qui supposent la triple obligation constitutive à la conception maussienne du don : donner, recevoir et rendre (Mauss 2007 [1925]). Comme la dévolution du crédit est échelonnée sur 25 semaines, elle doit être interprétée à la lumière d'une perspective faisant état de la dimension temporelle du don. Cette dimension articule non seulement la période au cours de laquelle se déroule le remboursement par étape des cotisations (prolongeant la relation de dette), mais aussi le système d'obligations qui précède la participation au programme de la BPBF et continuera d'exister par la suite.

Dans notre cas, les cinq membres du groupe solidaire sont obligés de recevoir, car si l'une d'entre eux ne prend pas le crédit, les autres ne peuvent pas le faire non plus. On pourrait donc considérer le microcrédit comme un type de transfert qui n'implique pas seulement des contreparties entre l'État et ses citoyens, mais aussi entre les bénéficiaires elles-mêmes. Chacune « offre » une garantie de solidarité au groupe. Elles « offrent» aussi leur temps en allant aux réunions et s'engagent à « rendre » l'argent reçu par des cotisations régulières. Il est ainsi possible de concevoir les microcrédits en termes de transfert (social), aussi bien qu'en termes de transaction (commercial). La relation constituée à partir de la dette qui fut initiée par le premier don (le crédit) est prolongée par la continuation de cette dette dans le temps. Or, cette relation montre aussi comment le rapport entre le donateur (l'État) et les donataires (les gens conçus comme étant pauvres) comportait déjà une dimension proprement relationnelle.

L'argent transféré par les microcrédits est le produit d'un travail social, c'està-dire qu'il découle du travail passé et actuel, formel et informel, redistribué sous la forme d'allocation ou de prestation. En d'autres termes, l'argent que le programme transfère fut généré, au premier lieu, par la population productive active et passive, puis, en second lieu, transféré à l'État qui, en troisième lieu, le redistribue à un segment de cette même population qui fut désigné comme «pauvre » par les autorités publiques. 
Or, ces transferts sont vécus de diverses manières par les bénéficiaires. Comme l'explique Guérin : « les femmes utilisent le microcrédit pour survivre, pour accumuler des liens de dettes et des rapports sociaux et pour négocier une meilleure position dans les espaces locaux de sociabilité » (Guérin 2014: 41). L'analyse anthropologique de ces « usages vécus » par les acteurs (Guérin 2015: 23) permet de connaître la manière dont le crédit et les rapports de dette qu'il mobilise sont saisis de manière native.

Dans ces échanges, les bénéficiaires sont recrutés pour participer à un cycle de «donner, recevoir, rendre » administré par l'État. En effet, en absence de conséquences économiques néfastes pour refuser de rendre, les bénéficiaires s'inscrivent clairement dans le jeu du don qui inclut par définition la « liberté » de « rebasculer dans le cycle négatif du prendre, refuser, garder» (Caillé 2006: 76-77). Les contrastes entre les cas de « succès » et « d'échec »dans l'application du microcrédit témoignent de la possibilité d'alterner entre ces cycles. Or, les cas d'échecs à l'étude montrent que les conditions qui déterminent la classification d'un échange ou d'une action comme appartenant au cycle «positif » ou «négatif » ne vont pas de soi et dépendent des relations à travers desquelles sont négociés les termes de la réciprocité attendue.

\section{LA FORMATION DU GROUPE «FLOR DE LOTO »: LA SOLIDARITÉ EN SEPT PAS ET AUTRES ÉLÉMENTS DE SUCCÈS}

L'objectif invoqué du programme de la BPBF est la diminution de la pauvreté à partir d'un système étendu de petits crédits octroyés à des personnes démunies qui se « connaissent mutuellement ${ }^{12}$ Ce crédit est ainsi demandé conjointement par cinq personnes qui s'engagent collectivement à rendre l'argent au banquito. L'idée est que ces relations personnelles réduiraient les risques associés à « prêter de l'argent aux pauvres ». La méthodologie envisage ainsi une « garantie solidaire » que l'on pourrait décrire comme étant morale plutôt que matérielle, c'est-à-dire qu'elle parie que la solidarité groupale peut agir comme mesure incitative pour assurer le remboursement. Comme condition pour pouvoir recevoir un microcrédit, chaque bénéficiaire doit présenter un projet (productif ou commercial), pouvant assurer leur « auto-emploi » et « améliorer ainsi leurs conditions de vie », selon la documentation de la BPBF (Conami 2010: 11). Or, chaque bénéficiaire donne aussi sa parole et fait de son honneur la caution de sa dette - l'octroi de prêts supplémentaires dépend du remboursement de l'ensemble des prêts du groupe solidaire.

Selon les documents officiels, la solidarité groupale commence avec la constitution du groupe pour demander le prêt. Cependant, il est clair que ces 
liens de solidarité reposent sur une vie de voisinage et des amitiés antérieures. La solidarité ne commence pas avec le microcrédit, mais beaucoup plus tôt, et doit être actualisée par un degré de leadership. En fait, le rôle de convocatrice d'Araceli fut très important pour la formation et les succès de son groupe. Tous les membres de son groupe se souviennent que c'était elle qui insistait pour qu'elles aillent au banquito. D'autres facteurs comme une disponibilité en matière de temps furent aussi soulignés pour expliquer leur participation et leur assiduité. L'une d'elles raconte par exemple : « elle m'a appelé, et m'a dit qu'elle passerait me chercher pour aller à une rencontre. Je n'avais pas envie, mais je n'avais rien à faire, c'était le week-end... et comme elle insistait et que sa maison est près de la mienne et que nous sommes voisines depuis 30 ans, j'y suis allée... » [Coca, 64 ans, cuisinière]. Les autres ont vécu des situations similaires. Marcela (35 ans, vendeuse d'essences aromatiques) se souvient qu'elle « s'ennuyait à la maison » quand elle a reçu un appel téléphonique d'Araceli. Chechu (73 ans, retraitée, artisan en bijouterie) se rappelle avoir croisé la belle-fille d'Araceli, Mica, à la pharmacie lorsque celle-ci lui a dit « qu'Araceli était à la recherche de personnes pour un projet... » Elle remarquait aussi l'importance du temps libre dans sa décision : après « je me suis joint au projet, comme je suis à la retraite j'ai beaucoup de temps et j'aime bien sortir, et ne pas rester chez moi... ». Mica (22 ans, vendeuse de vêtements), pour sa part, explique qu'Araceli était toujours chez elle, car Mica est «mariée avec le fils d'Araceli et habite dans une petite maison construite à côté de la sienne». Elle a donc « conçu un projet pour l'aider à former le groupe ».

Les témoignages des participantes signalent que vivre à proximité et avoir du temps libre constituaient de bonnes raisons à se rejoindre au programme. Mais le fait qu'Araceli les emmenait aux réunions dans sa voiture a aussi grandement contribué à l'assiduité des membres de ce groupe. En fait, personne n'a jamais manqué une réunion, mais les participantes admettent que sans ces voyages gratuits et l'insistance de leur voisine, elles ne seraient probablement pas allées aux réunions chaque semaine et auraient peut-être quitté le programme : «Araceli téléphonait toujours et demandait si tu avais l'argent ou si tu avais besoin qu'elle t'en prête, cela te mettait de la pression et je pense que pour ça nous allions bien au banquito » [Coca].

Au cours de ces semaines, chaque groupe solidaire doit se donner un nom. Le groupe d'Araceli s'est donné le nom «Flor de Loto» (Fleur de Lotus), en s'inspirant des valeurs de la paix et de l'équilibre que ce symbole incarne. Cela reflète l'importance du projet de son leader (la revente de vêtements venant d'Inde) et son rôle dans la construction de l'identité du groupe. Le leadership d'Araceli et la disponibilité de ses partenaires ont contribué à la capacité de ce groupe à incarner un cas de succès aux yeux du programme, non seulement parce que l'ensemble de ses membres ont su rembourser leur prêt, mais aussi parce qu'ils ont satisfait au critère de solidarité mis de l'avant par les 
administrateurs du microcrédit. Aux yeux des promoteurs, c'est le groupe qui garantit que les dettes soient honorées et non pas les individus. D'où l'importance de la solidarité et notamment de la « garantie solidaire » dans la BPBF, qui opère ainsi selon des principes de collaboration économique semblable au modèle de la tontine. ${ }^{13}$ Il est entendu que cette cohésion groupale soit cimentée par divers éléments. Pour que la solidarité fonctionne, les membres d'un groupe sont sélectionnés par leurs pairs et doivent s'y joindre sans coercition externe. Pour éviter la désertion, les crédits sont versés de manière échelonnée et les remboursements sont demandés à des intervalles fréquents (hebdomadaires, bihebdomadaires ou mensuels). La possibilité de renouveler la demande de crédits pour des montants chaque fois plus grands est ensuite perçue comme une mesure incitative pour encourager le remboursement et la responsabilité des membres envers leur groupe. Ces principes se réalisent dans un cycle qui comporte plusieurs étapes : (1) la constitution de l'équipe de promotion ; (2) la formation des promoteurs ; (3) le recrutement des participants ; (4) la constitution des groupes ; (5) le programme « des sept pas » qui, au cours de sept semaines, vise à préparer les dossiers de demandes individuels et consolider l'identité groupale ; (6) l'octroi des crédits ; (7) le remboursement du prêt et la participation des groupes de bénéficiaires à la vie collective du banquito locale ; et (8) l'octroi d'un deuxième crédit (le « recrédit »). La méthodologie Grameen envisage ainsi un processus de consolidation des relations de solidarité allant des premiers liens de confiance jusqu'à leur parachèvement après la réalisation des «sept pas ». Or, elle imagine aussi une solidarité plus large, et sur le plus long terme, alimentée par le recrédit et la participation au développement de la communauté des banquitos.

Les promoteurs retrouvent cet itinéraire sous forme écrite dans le manuel de travail de la BPBF confectionné par l'équipe technique du Ministère du développement social et intitulé «L'économie sociale et l'éducation populaire » (Conami 2010). Ce document résume les lignes directrices du programme de microcrédit, mais il s'exprime aussi dans un langage qui valorise les fonctions sociales du gouvernement national. Il demeure un manuel pratique - un outil de travail pour la constitution des groupes solidaires - et offre un modèle idéal pour que chaque banquito adapte la méthodologie Grameen aux conditions locales. Mais le manuel assume aussi un rôle idéologique dans la mesure qu'il transmet les clés d'interprétation de l'esprit du programme. Le banquito y apparaît comme une «maison que l'on construit ensemble », comme une banque de type «non-commercial » et comme les ingrédients d'un « grand réseau national et populaire » qui coïncide avec la «Patrie ». La BPBF y est décrite comme étant

13 La tontine est une association collective d'épargne qui réunit des épargnants pour investir en commun dans un actif financier ou dans un bien, de sorte que le projet dans son ensemble dépend de la participation collective. 
« participative » et fondée sur les valeurs de «l'honnêteté », de la "solidarité », de la «confiance » et de «l'engagement». Pour ce qui est des bénéficiaires, ils sont les «protagonistes » qui constitueront « les bases populaires » d'une société qui souscrit à des «valeurs éthiques et solidaires » par opposition à celles du «marché ». Les promoteurs investissent aussi ce prosélytisme. Ils expliquent à chaque candidat que pour faire partie de la " petite banque », il faut « participer » aux réunions hebdomadaires, adhérer à « certaines valeurs » et surtout « être solidaire». C'est dire que le programme vise le développement d'une économie dite "sociale », et propose, de sorte, des dynamiques de fonctionnement de type groupal. En somme, il dit vouloir non seulement à générer des emplois, mais aussi des « rapports de solidarité », voire un projet « national et populaire » qui se fonde « à partir du bas ».

L'observation des interactions entre les promoteurs et les bénéficiaires indique que le répertoire symbolique de cette "solidarité » (de groupe) est systématiquement lié à celui de la confiance en soi (individuel). À suivre la logique des promoteurs, le répertoire de l'estime de soi complète la pratique collective de la constitution du groupe solidaire. En fait, après avoir sollicité et reçu le crédit, les femmes sont instruites sur la valeur de la confiance en soi :

« Recevoir le crédit exige que vous fréquentiez beaucoup de gens... vous devez quitter votre maison à la recherche de personnes qui voudraient devenir vos partenaires. À cette fin, vous devez vous fier à vos propres moyens, penser que cela vaut la peine, et que d'ailleurs vous êtes en mesure de bien le faire. Au fur et à mesure que l'on avance et que l'on commence à avoir du succès (on obtient le crédit, des matériaux, des contacts, des acheteurs de produits), l'estime en soi augmente parce qu'on se sent bien avec soi -même» [promotrice, 42 ans, vendeuse].

Ces mots d'une bénéficiaire qui avec le temps est devenue promotrice sont souvent utilisés pour mettre en relief les effets de l'autonomisation que génère le microcrédit. L'autosuffisance est ici associée à une vision entrepreneuriale de l'estime de soi que l'on espère cultiver de concert avec le développement de comportements « solidaires ». Selon les promoteurs, cette confiance augmente en participant et s'engageant dans les activités du banquito : tenir sa parole, ne pas disparaître et toujours aller aux réunions sont des activités qui articulent la confiance en soi et la confiance dans le programme. Dans cette perspective, la solidarité est avancée comme un juste moyen pour que les bénéficiaires se transforment en femmes qui réussissent à l'auto-emploi. Malgré le fait que leur succès semble reposer sur des ressources et une solidarité qui précédaient leur participation au programme, le groupe d'Araceli fut considéré comme un exemple de succès de celui-ci, principalement dans la mesure qu'il a pu 
satisfaire les obligations qui lui furent imposées. « L'insuccès » et «la non-solidarité », à ce titre, sont définis par opposition à cette conformité.

«LES PERSÉVÉRANTES » ET LEUR «INSUCCÈS » DANS LE PROGRAMME : COMPLOT ET MANQUE DU TEMPS

Le nom «Les Persévérantes » fut choisi par Marie et ses collaboratrices pour décrire leur groupe. Par cela, elles cherchent à évoquer la force de volonté de celles qui avaient déjà essayé de demander des crédits sans succès. De leur point de vue, la volonté et la persévérance étaient ce qui les définissait le plus, car elles avaient pu supporter le manque de financement. Elles expliquent la chose ainsi :

« quand nous réussissions à convoquer des gens et organiser un groupe, l'argent n'arrivait pas [c'est-à-dire, que l'État ne disposait pas de fonds suffisants], et quand finalement les banquitos réussissaient à obtenir des virements, les groupes n'existaient plus, car les gens s'étaient dispersés pendant la longue attente » [Marie, 45 ans, coiffeuse].

«Les Persévérantes » ne purent cependant imiter les «succès » de «Flor de Loto ». Trois semaines après l'octroi du crédit, deux « persévérantes » avaient déjà cessé d'assister aux réunions et interrompu leurs remboursements. Entre la huitième et la dixième semaine, deux autres avaient déserté leur bande. La désintégration graduelle du groupe fut ensuite suivie par sa dissolution finale. En refusant de continuer à donner leur temps au programme et en percevant leur crédit sans le rembourser, ces bénéficiaires s'engagent dans des pratiques de prendre-garder-refuser qui rompent avec les attentes du donner-recevoir-rendre, mais constituent en fait toujours un débouché possible de la dynamique du don ( $c f$. supra). Pour les promoteurs qui avaient participé à la formation de ce groupe et au renforcement de la solidarité entre ses membres, il s'agit d'une infidélité qui va au-delà de la transgression d'une attente, car elle encourt le risque que le banquito soit mal évaluée par le ministère. Les coordinateurs, pour leur part, supposent que cet échec résulte de dérogations dans la mise en œuvre de la méthodologie. Mais en quoi consiste vraiment le contraste entre le cas des Persévérantes et celui de Flor de Loto ?

Du point de vue des règles du programme, cette expérience est un « cas d'échec » pour différentes raisons. Premièrement, le crédit ne fut pas remboursé dans son entier. Une seule des membres a versé l'ensemble de ses remboursements. Deux autres n'ont que partiellement remboursé leur prêt et le reste n'a rien remboursé et a quitté le programme immédiatement après avoir reçu le crédit. Deuxièmement, la participation au programme n'a guère amélioré les conditions de vie des bénéficiaires, qui ont continué avec leurs activités 
initiales : femmes au foyer (dans tous les cas), coiffeuses, couturières, cuisinières de milanesas, ${ }^{14}$ marchandes de légumes, brodeuses et femme de ménage. En troisième lieu, dans certains cas, l'activité productive à laquelle le crédit devait être destiné n'a jamais été entreprise. Du point de vue des promoteurs, ces femmes les avaient trompés en simulant une bonne foi qu'elles n'avaient pas, et en feignant une solidarité qu'elles ne pratiquaient pas authentiquement; le problème était réductible à un manque d'engagement avec la logique solidaire que proposait la BPBF. En d'autres mots, les bénéficiaires n'avaient pas développé « le comportement économique et solidaire » que proposait le programme.

Par contre, du point de vue des bénéficiaires, ce qui s'était passé fut normal en partie par cause de l'insuffisance des montants octroyés : «le crédit ne suffit à rien... Il est fou d'espérer qu'on puisse faire grand-chose avec si peu d'argent », dit Marita (38 ans, employée domestique). Les 900 pesos octroyés « glissent comme de l'eau entre les mains », ajoute-t-elle, et « suffisent à peine pour vivre au jour le jour pendant peu de semaines $» .{ }^{15}$ Les bénéficiaires de ce groupe ont aussi œuvré à redéfinir les objectifs originels du prêt. Ce faisant, elles affirmèrent non seulement que le crédit n'avait pas besoin d'être destiné aux activités productives qu'elles avaient déclarées dans leur « dossier d'entrepreneur », mais aussi que l'accomplissement des obligations de remboursement pouvait être conditionnel à d'autres engagements ou priorités. Certains m’ont confessé que dans leur vie quotidienne elles utilisaient le crédit surtout pour « acheter de la nourriture et pour payer des impôts », au lieu d'investir dans les activités de production. Par exemple, avec l'argent reçu, Marita a payé une dette accumulée dans un magasin et a acheté des vêtements pour son fils de 12 ans. Elsa (52 ans, cuisinière) m'a avoué qu'au lieu d'investir son prêt dans l'achat d'un ensemble de couteaux pour cuisiner, comme elle l'avait proposé dans son dossier, elle l'a utilisé, en réalité, pour réparer une fenêtre dans sa maison. Marie, pour sa part, n'a pas utilisé le crédit pour améliorer son salon de coiffure, mais pour payer des comptes dans un magasin. Elle s'est justifiée en disant qu'elle « avait déjà perdu tant de temps avec l'autre appel de crédits, j'avais besoin de faire tout express... Alors, j'ai menti un peu... Je m'en fous du salon ».

En effet, le processus de formation du groupe a été marqué par les difficultés de Marie. Une de membres se rappelle que Marie l'a appelé, «à l'époque où elle était en train de se divorcer et elle avait toujours des problèmes avec son mari, son fils... Elle me parlait de la petite banque... Moi, je ne voulais pas

14 Milanesas c'est l'un des plats les plus populaires du pays.

15 Dans l'Argentine de 2011 , une somme de 900 pesos représentait 39\% d'un salaire minimum par mois (fixé à 2300 pesos ou 518 dollars) et $18 \%$ d'un salaire moyen par mois (5000 pesos ou 1126 dollars). 
y participer, mais je lui ai dit d'y aller, parce qu'avec ses problèmes familiaux elle avait vraiment besoin de l'argent » [Norma, 48 ans, couturière]. À cause de sa récente rupture, Marie ne pouvait pas assister aux réunions ni avertir ses camarades de payer leurs cotisations. De plus, ses camarades se rappellent qu'ils ont «vu Marie très peu ». Trois d'entre elles, Elsa, Marita et Patricia, disent qu'elles ont « seulement parlé » à Marie au moment qu'elle les a contactées pour joindre le groupe et qu'« après elle a disparu ». Seule Norma avait une relation étroite avec Marie qui a continué après la dissolution du groupe. Marie, pour sa part, disait qu'elle travaillait du matin jusqu'au soir et qu'elle n'avait plus de temps pour le banquito.

Pour les promoteurs, ce cas démontre les effets délétères de comportements « non solidaires ». Or, certaines observations suggèrent que ces pratiques renvoient à une « solidarité » de la « complicité » différente de la « solidarité standardisée » que la méthodologie Grameen cherche à exporter. Par exemple, l'une des promotrices a découvert que les deux premières femmes à recevoir leur crédit étaient amies et avaient « comploté pour que l'une d'elles s'approprie l'argent des deux avant de disparaître ensemble ». La seule femme du groupe qui avait payé la totalité de son crédit m'a raconté d'un air contrarié et de manière confidentielle que « tout était arrangé ainsi dès le commencement » entre ces deux complices : «elles savaient que si l'on ne payait pas il n'y avait pas du tout de conséquences, qu'ils ne pouvaient rien te faire; de ce fait, elles ont tout préparé de manière secrète, elles ont parcouru les sept pas comme un groupe solidaire, elles ont joué comme dans un théâtre, exactement jusqu'au moment où elles ont touché l'argent et elles sont immédiatement parties ». Apparemment, l'instigatrice, Marie, et sa complice, Norma, avaient commencé un cycle inverse à celui du don maussien où elles avaient décidé prendre les crédits, refuser de continuer leur participation au banquito et garder l'argent en refusant de le rendre.

Cependant, en approfondissant l'analyse sur les justifications natives de l'inaccomplissement de la dette, on observe que le manque de sanction et la quantité excessive du temps investi sont des éléments importants dans les discours des bénéficiaires qui n'ont pas rendu du crédit. À plusieurs occasions, les femmes ont dit qu'elles ne rembourseraient pas le prêt parce que « rien ne se passe »; c'est-à-dire parce qu'il n'y a pas de punition directe pour cette transgression à la norme de «tenir sa parole » que les promoteurs ont tenté d'intimer dès le début. Mais il y a encore une deuxième justification : la volonté de substituer l'argent par le temps. Les femmes expliquent qu'elles « ne peuvent plus aller aux réunions parce qu'elles n'ont pas de temps », ou bien qu'elles ont « accompli toutes les heures gaspillées durant toutes ces réunions pour huit mois! Pour ne recevoir que 900 pesos !» De cette manière, elles dévaluent l'importance du montant versé et laissent entendre qu'elles l'ont déjà remboursé avec le temps de participation destiné au programme. 
Cette interprétation native de la dette est en concordance partielle avec ce que le programme demande à ses participants, en attendant non seulement le remboursement de la dette, mais aussi une contre-prestation dénommée «participation et engagement ». Néanmoins, le programme n'envisage pas cet échange comme l'expression d'un comportement solidaire.

Les rhétoriques justificatrices du non-remboursement de la dette viennent ainsi appuyer des pratiques qui correspondent à une espèce d'accord derrière le dos du banquito, s'opposant à la forme de solidarité standardisée avancée par le programme. Quitter le programme est considéré dans cette perspective comme une action juste par rapport à ce qui a déjà été donné. Du point de vue des bénéficiaires, la dette est soldée. Comme l'explique l'une des «persévérantes » :

«Ils nous ont dit d'avoir confiance en nous-mêmes, mais ça ne suffit pas... parce que si vous avez une forte estime de soi, mais que vous ne vendez rien, d'où sort l'argent ?! En principe nous allions toujours avec Elsa [une autre collègue du groupe solidaire] aux réunions et nous payions chaque semaine la cotisation... Mais après les quatre premières fois, on s'est rendu compte que la seule chose qui intéressait les promoteurs c'était de toucher l'argent. Ils ne s'intéressaient pas à savoir si on avait vendu ou pas, si nous avions des difficultés... Ils ne nous demandaient rien... Nous avons cessé d'y aller. Je me souviens que nous étions chez Elsa et quelqu'une a dit : 'pourquoi aller gaspiller notre temps à ces réunions juste pour avoir reçu cet argent de misère après tout le temps qu'on n'y a déjà dépensé', et voilà, on n'y est plus jamais allées » [Norma, 48 ans, couturière].

Ces témoignages suggèrent que la décision de quitter le programme est liée non seulement au type de leadership développé par Marie, mais aussi à la représentation pratique de la dette remboursée. Ces représentations sont liées aux ressources monétaires disponibles ou non pour rendre le crédit et à la valorisation positive de la principale ressource déjà donnée au programme : le temps.

\section{LA VALORISATION NATIVE DU TEMPS COMME RECOURS DE SUBSTITUTION AU REMBOURSEMENT DE DETTES}

Les comportements des deux groupes étudiés étaient visiblement très différents. Dans le cas de «Flor de Loto », Araceli s'occupait dès le début du ralliement de ses camarades en les convainquant de demander le crédit et d'offrir leur propre maison pour tenir les réunions de groupe. Elle conduisait ses collègues pour payer leurs cotisations et leur prêtait de l'argent si elles ne pouvaient pas le faire. En contraste, la leader des « Persévérantes » n'avait pas cette sorte de disponibilité. Alors que les membres du groupe d'Araceli se 
réunissaient fréquemment pour boire du mate, ${ }^{16}$ s'échangeaient des services et des faveurs, se conduisaient mutuellement aux réunions et s'appelaient par téléphone afin de coordonner des activités, le groupe de Marie ne faisait que le minimum demandé par le manuel par rapport à la cohésion groupale. On pourrait penser que «Les Persévérantes » ont «échoué » à cause d'un vide de leadership. Aux yeux de ses camarades, Araceli avait ce qu'en espagnol on appelle don de gente (en français : le savoir de s'y prendre avec les gens, être de bon commerce). Marie, pour sa part, semblait « ne penser qu'à l'argent ». D'une certaine manière, la première, par son caractère agréable et ses propres recours, contribuait à maintenir le groupe uni et à payer les dettes à temps. En revanche, l'urgence et le manque de recours ont fini par dissoudre le groupe de la deuxième.

Les justifications natives des pratiques de prendre-garder et de refuser de rendre attire par contre l'attention au fait que les bénéficiaires évoquent le temps lorsqu'elles sont invitées à expliquer leurs relations à la dette non remboursée. Dans ces situations, leur répertoire ne reprend pas les catégories des promoteurs - tels que l'inaccomplissement, le mensonge, la fausse solidarité, le manque d'engagement, le refus de tenir sa parole - mais plutôt des thèmes exprimant leur désir de récupérer le temps perdu : «on ne peut pas continuer à perdre notre temps à ses réunions avec tant de choses à faire à la maison ». Ainsi, explique l'une des bénéficiaires :

« J'ai enduré tout cela, les réunions et le fait de remplir plusieurs formulaires, malgré la grande quantité de choses que j'avais à faire, seulement en considération de mes partenaires. Mon Dieu ! Je ne voulais pas quitter le groupe après tant d'efforts. Mais ils nous demandent d'y aller presque pendant deux mois, juste pour remplir des formulaires et pour ne nous donner que 900 pesos! Que peut-on faire avec 900 pesos ?! Je connais d'autres personnes qui étaient également fatiguées et qui ne voulaient pas aller aux réunions, mais elles ont enduré tout ça parce qu'elles avaient besoin de l'argent et parce qu'elles savaient que les autres en avaient aussi besoin » [Marie].

D'autres membres du groupe utilisent le départ de Marie pour justifier leur abandon du programme :

« Moi au début j'y allais toujours pour payer la dette, mais après Marie n’y allait plus et puis je me suis informée que si tu ne payes pas il ne se passe rien. C'est pour cela que moi non plus je n'y suis plus allée... Mais surtout à cause du manque de temps, pas pour ne pas rendre l'argent... mais bon,

16 Le mate est une infusion typiquement argentine, bue fréquemment à la maison avec la famille et les amis. 
qu'est-ce que je fais ? C'est compliqué pour moi d'y aller, je n'ai pas de temps » [Patricia, 38 ans, artisan].

Par rapport au non-remboursement, elles se sentent satisfaites d'avoir soustrait quelque chose du programme (Marita), car de cette façon au moins, elles n'auront pas perdu tant de temps pour si peu d'argent (Norma).

À force d'écouter les participantes de « la petite banque » se plaindre de la quantité excessive de temps nécessaire à solliciter le microcrédit, je me suis mis, en tant qu'analyste, à considérer leurs plaintes comme dénotant une valorisation positive de cet élément à titre de ressource. Cette valorisation native, qui devient visible grâce à l'analyse anthropologique de la dimension vécue de la dette, est mobilisée par les acteurs pour prendre des décisions, mais aussi pour construire des justifications. Elle met aussi en lumière les représentations natives des rapports sociaux qui sous-tendent la dette, aussi bien entre pairs qu'envers l'État. D’un point de vue strictement économique, le crédit correspond à une transaction monétaire entre deux agents comprenant un montant et un taux d'intérêt pour un délai de remboursement accordé. Selon ce schéma économiciste, quand la dette est payée dans le temps accordé, la relation économique est considérée comme étant résolue.

Or, du point de vue anthropologique, les choses données et ensuite dues n'ont pas une définition unilatérale ou universelle et l'échange ne peut être abstrait des relations concrètes qui la constituent. Dans le cas étudié, la valorisation native du temps substitue l'argent dans les répertoires sur les obligations acquises par le crédit. Par ailleurs, du point de vue de la structuration des rapports de dettes, cette perspective native semble adopter une forme de solidarité qui infléchit celle envisagée par le programme. Dans certains cas, les bénéficiaires privilégient des formes de solidarité - de voisinage ou de complicité - qui précèdent celle qu'impose le programme. Or, plus fondamentalement, les débiteuses dissidentes cherchent à renégocier les termes de la solidarité du programme en construisant une symétrie horizontale entre l'argent reçu et le temps dépensé. Le temps qu'elles ont donné leur permet d'argumenter en faveur d'une définition différente des obligations qu'elles ont accrues en acceptant le crédit, c'est-à-dire une définition plus avantageuse vu leur situation particulière. De cette façon, les bénéficiaires atténuent la dépendance générée par la verticalité asymétrique du transfert étatique.

Les aspects interpersonnels de la dynamique du microcrédit montrent qu'au niveau local, le microcrédit se comporte comme un système complexe de transferts simples (le don) et de doubles transferts différés (le contre-don). Or, l'expérience de la dette peut produire une grande variété de sentiments et de significations dépendamment du sens accordé au don et de la nature de la relation entre le donateur et celui qui reçoit le don. Le résultat du don peut être la cohésion sociale et la solidarité, mais aussi l'asymétrie et la domination 
(Mauss 2007 [1925]). Dans ces échanges, la décision de ne pas rendre l'argent du crédit et de le garder n'est pas tant le résultat de dispositions non solidaires que d'un effort de redéfinir les termes de la relation de don. Ainsi, les bénéficiaires utilisent le temps donné au programme pour rembourser la dette monétaire avec les ressources dont elles disposent et dont elles ressentent aussi la rareté. En octroyant une valeur à leur temps, elles cherchent à reformuler les termes du donner-recevoir-rendre pour qu'ils incluent leurs pratiques de prendre-garder-refuser. De ce fait, elles proposent une solidarité basée sur l'échange de ressources dont elles disposent effectivement et ont pu en effet contribuer. Que l'État accepte ou non cette redéfinition ne change pas le fait qu'il pourrait, en principe, le faire et de ce fait réinscrire le refus de rendre dans la dialectique du don.

\section{CONCLUSION}

Dans le contexte des politiques publiques qui cherchent à aborder la pauvreté, je me suis proposé ici de comprendre l'usage que les acteurs font des notions de dette et de solidarité. Pour cela, je les ai analysées comme des représentations de transferts s'inscrivant dans le registre du don-contre-don.

Mon intérêt pour l'expérience vécue du microcrédit m'a amené à enquêter sur les relations d'endettement dans les banquitos argentins. En cherchant à comprendre comment le programme pense pouvoir assurer le remboursement des prêts qu'il octroie sans autres garanties que la parole des récipiendaires, j'ai aussi découvert l'existence de mécanismes de dissolution de dettes parallèles dans les répertoires des participantes. Certaines ont mis en profit leur temps libre et leur solidarité de voisinage pour arrimer leur comportement aux desiderata de la méthodologie Grameen coordonnée par les promoteurs, soit « les sept pas ». Mais d'autres bénéficiaires ont comploté pour toucher à l'argent et quitter le programme ou ont jugé que la part de leur temps capturé par le programme constituait un remboursement adéquat de la modeste somme octroyé.

Les acteurs assemblent leurs groupes solidaires selon deux tendances : une tendance qui exprime des raisons de caractère social, associées au loisir et à la proximité (surtout, elles veulent échapper à l'ennuie et aider Araceli, qu'elles connaissent depuis longtemps) ; une autre tendance qui exprime des raisons de caractère économique, moins associées aux liens de proximité et disposant de moyens limités pour maintenir le groupe uni. Cela ne veut pas dire que «Les Persévérantes » sont « moins solidaires » que « Flor de Loto » et que c'est pour cette raison qu'elles ont échoué aux yeux du banquito. Après l'analyse, je suis incliné à penser que les bénéficiaires qui ont connu un succès dans le programme - c'est-à-dire celles qui peuvent effectivement rendre le crédit et obtenir des crédits supplémentaires - sont des personnes qui avaient des 
recours suffisants et qui n'avaient pas tant besoin de secours financiers dans leurs vies quotidiennes.

La « solidarité standardisée » cultivée par « les sept pas» génère une normalisation des transactions et transferts qui s'arriment à la définition du « solidaire » conforme aux règles du programme. Dans ce contexte, on fait abstraction des recours sociaux et économiques qui sont préalables et complémentaires à ceux de la participation au programme. Cette définition de la solidarité ignore les ressources matérielles qui assurent la formation d'un groupe solidaire performant. Les cas de « Flor de Loto » et des « Persévérantes » soulignent l'importance de ces ressources dans le développement de la «solidarité » envisagé par le programme et dans le succès ou insuccès de la méthodologie. Les membres du groupe qui n'ont pas remboursé leur prêt ont utilisé l'argent perçu pour des nécessités domestiques au lieu d'atteindre les objectifs entrepreneuriaux présentés dans leurs dossiers.

Par ailleurs, la valorisation que les acteurs produisent du donné et du dû dans le processus d'acquisition, de dévolution et de non dévolution du microcrédit demande de connaître les différentes perspectives natives impliquées dans la construction de la solidarité. En comprenant d'un point de vue ethnographique les rapports de dette mis en place par les programmes de microcrédit, j'ai utilisé une notion du don qui tient en compte certaines expériences de refus de rendre. En ce sens, j'affirme que certains comportements considérés comme étant non-solidaires, comme prendre, garder et refuser le crédit, font aussi partie du phénomène du don. Dans le répertoire des promoteurs, garder ou ne pas rendre l'argent reçu implique une sorte de trahison ou de faillite. Mais du point de vue analytique développé ici, érigé sur les perspectives natives des cas d'échec, ces pratiques composent avec une valorisation du temps qui cherche à renégocier les termes du contre-don.

La perspective des « sept pas » standardisés mises en œuvre par les promoteurs et les prestataires « exemplaires » contraste avec la perspective de ceux qui furent caractérisés de «mauvais exemples » de solidarité. En d'autres mots, certaines bénéficiaires n'ont pas pu adapter leurs comportements aux attentes du type de solidarité que le programme cherche à développer. Le travail de terrain indique qu'il existe des éléments extra-bancaires qui conditionnent le succès ou l'échec des activités des banquitos. Les facteurs économiques sociaux qui peuvent servir à expliquer le succès et l'échec sont opacifiés dans les répertoires des intervenants de l'État, mais aussi dans ceux des bénéficiaires. En fait, la pratique de substituer l'argent par le temps pour régler la dette éclipse de manière similaire l'indisponibilité général des ressources pour rembourser le prêt. Cependant, elle met aussi en lumière un autre aspect : celui de la valorisation native du temps dans l'optique de redéfinir les termes du contredon. Loin de critiquer la bonne volonté du programme, j'ai voulu, par ce travail de terrain, contribuer à penser sa complexité, malheureusement souvent 
simplifiée par deux types de naturalisations dans l'application argentine de la méthodologie Grameen : (1) une vision homogène des conditions locales et (2) le sens univoque de la «solidarité » proposée par le programme. Sans verser dans un relativisme radical, je pense qu'il faut cesser de penser reconnaître «La Solidarité » (avec une majuscule) et nous occuper à comprendre les formes particulières de solidarité et de mutualisme. Plus encore, il serait convenable d'explorer les conditions - par exemple verticalité, asymétrie, précarité - qui pousse aux récipiendaires d'un crédit à vouloir renégocier les termes de son remboursement. Dans ce sens, le fait de ne pas rendre le crédit, mais de rester dans une chaîne de faveurs entre voisines pour qu'elles puissent aussi percevoir leur prêt, se présente comme une transformation du crédit en un don dont le contre-don est attendu, mais susceptible de prendre une forme qui n'est pas définie d'avance. En invoquant le « manque de temps pour aller à tant de réunions », les femmes construisent des rapports de dettes réciproques qui leur permettent de renégocier leur position d'acteurs endettés en mettant en valeur leur participation.

\section{BIBLIOGRAPHIE}

AHNEN, Ronald, 2017, "Leftist politics and the limits of microcredit in Argentina", Journal of Politics in Latin America, 9 (2): 41-62.

BOURDIEU, Pierre, 2003 [1967], "La fabrique de l'habitus économique", Actes de la recherche en sciences sociales, 150: 79-90.

CAILLÉ, Alain, 2006, "Apologie et critique du don : le don entre science sociale et psychanalyse, l'héritage de Mauss jusqu'à Lacan”, Revue du MAUSS, 27: 57-78, DOI: 10.3917/ rdm.027.0057.

CONAMI - COMISIÓN NACIONAL DE MICROCRÉDITO, 2010, Banco Popular de la Buena Fe: Manual de Trabajo. Buenos Aires, Ministerio de Desarrollo Social de la Nación.

FOUILLET, Cyril, et al., 2007, "Le microcrédit au péril du néolibéralisme et de marchands d'illusions: manifeste pour une inclusion financière socialement responsable", Revue du MAUSS, 29: 329-350, DOI: 10.3917/rdm.029.0329.

GUÉRIN, Isabelle, 2014, "Juggling with debt, social ties, and values: the everyday use of microcredit in rural South India”, Current Anthropology, 55 (9): 40-50.

GUÉRIN, Isabelle, 2015, La microfinance et ses dérives : Emanciper, discipliner ou exploiter? Paris, Demopolis.

GUÉRIN, Isabelle, et Jean Michel SERVET, 2005, "Léconomie solidaire entre le local et le global : l'exemple de la microfinance”, Revue internationale de l'économie sociale, 296: 83-99. GUÉRIN, Isabelle, Marc LABIE, et Jean-Michel SERVET (orgs.), 2015, The Crises of Microcredit. Londres, Zed Books. 
KOBERWEIN, Adrián, 2011 , "Consumo y dinero: transacciones legítimas, acuerdos y conflictos interpersonales en un programa de microcréditos”, Runa, 32 (2): 185-202.

KOBERWeIN, Adrián, 2012, Microcrédito, Relaciones Personalizadas, Economía y Política: El Crédito para los Pobres, de Bangladesh a la Argentina. Buenos Aires, Antropofagia.

MAUSS, Marcel, 2007 [1925], Essai sur le don. Paris, Presses Universitaires de France.

MORVANT-ROUX, Solène, et al., 2014, "Adding value to randomization with qualitative analysis: the case of microcredit in rural Morocco", World Development, 56: 302-312.

SAIAG, Hadrien, 2011, “¿Monedas para el cambio social? Algunas reflexiones acerca del trueque Argentino", dans R. Orzi (org.), Moneda Social y Mercados Solidarios II: La Moneda como Lazo Social. CCCUS, Buenos Aires, 205-235.

SCHIJMAN, Emilia, 2014, "La vie privée des chômeuses", Esprit, 11 (2014): 38-46.

SERVET, Jean-Michel, 2006, Banquiers aux pieds nus : la microfinace. Paris, Odile Jacob.

WEBER, Florence, 2007, "Vers une ethnographie des prestations sans marché", préface à M. Mauss, Essai sur le don. Paris, Presses Universitaires de France, 7-62. 